\title{
A systematic review on radiographers' knowledge in imaging
}

\begin{abstract}
Background: Diagnostic imaging is one of the main functions of a Radiology Department. It needs a knowledgeable practitioner to ensure the image quality and ultimately, stakeholders' satisfaction. Objective: The aim of the study is to review scientific articles that discuss radiographers' knowledge in imaging and factors associated with the radiographers' knowledge. The review also identified the types of intervention which has been done to increase the radiographers' knowledge on imaging. Methods: A systematic search was performed by using the following electronic search engine: Pub-med, Science Direct, Medline and CINAHL. "Radiographer" and "knowledge" are the specific keywords that were utilized in the search on title, abstract and keyword. Articles included in the current study were subjected to specific criteria. The inclusion criteria are all studies that were published until December 2016, written in English, conducted on different diagnostic imaging sections and studies that evaluate radiographers' knowledge. Studies that were excluded include those that were conducted only partly in radiology sciences and studies that measured the equipment techniques. Results: A total of 28 articles were identified, of which, only fifteen studies met the inclusion and exclusion criteria, and therefore eligible to be included. Conclusion: The selected studies emphasized on training or education programs to improve respondents' knowledge and to improve imaging process and reduce potential imaging error.
\end{abstract}

Keyword: Associated factors; Imaging; Intervention education; Knowledge; Radiographers 